NISSUNA UMANA INVESTIGAZIONE SI PUO DIMANDARE VERA SCIENZIA S'ESSA NON PASSA PER LE MATEMATICHE DIMOSTRAZIONI LEONARDO DA VINCI

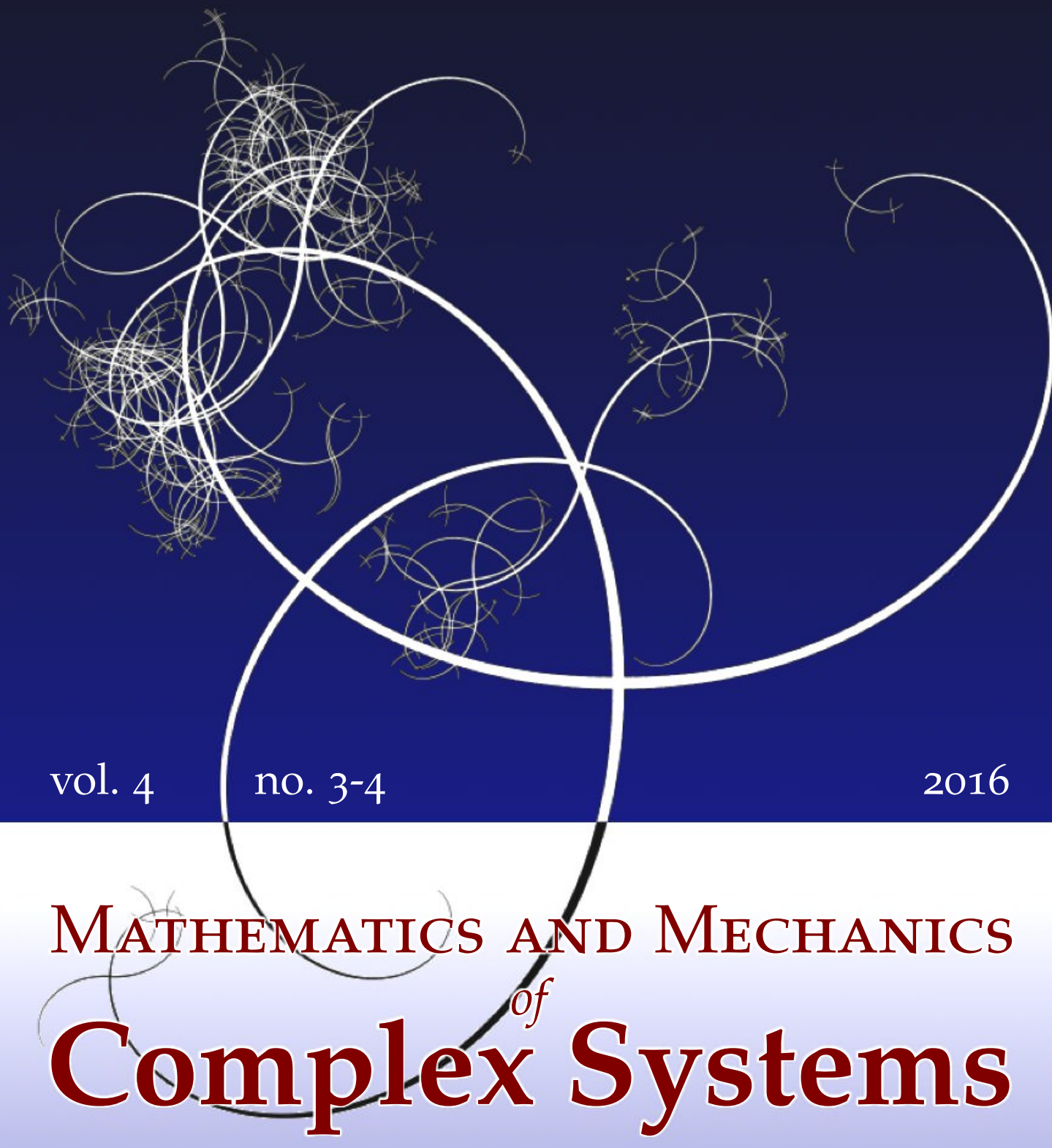

Christian Maes

WHAT DECIDES THE DIRECTION OF A CURRENT? 


\title{
WHAT DECIDES THE DIRECTION OF A CURRENT?
}

\author{
Christian MAES
}

Dedicated in honor of Lucio Russo

\begin{abstract}
Nonequilibria show currents that are maintained as the result of steady driving. We ask here what decides their direction. It is not only the second law or the positivity of the entropy production; nondissipative aspects also often matter and sometimes completely decide the direction.
\end{abstract}

1. Introduction

2. Traditional arguments 277

3. Problematic cases 282

4. Low temperature analysis 290

5. Conclusion 294

Acknowledgment 294

References 294

\section{Introduction}

Predicting the course of events given the present state is part of scientific practice. In what direction things will evolve is however not always so evident. In thermodynamics, there are a number of general rules of thumb derived from the principal laws. For instance, macroscopic systems tend to equilibrate at the same temperature, chemical potential and pressure as the surroundings, relaxation (or time itself) flows in the direction of increasing entropy, etc. In mechanics, the ambition is even higher; we compute trajectories given the present state. Statistical mechanics is supposed to transfer mechanical laws to thermodynamic behavior, with the attenuation that some thermodynamic principles are not absolute but become statistical. For example, the Boltzmann equation for a dilute gas has a direction of time, but for mesoscopic systems, fluctuations can be expected, and as Maxwell emphasized,

\section{Communicated by Raffaele Esposito.}

MSC2010: 82C03.

Keywords: direction current, nonequilibrium statistical mechanics. 
"The truth of the second law is ... a statistical, not a mathematical, truth, for it depends on the fact that the bodies we deal with consist of millions of molecules ... Hence the second law of thermodynamics is continually being violated, and that to a considerable extent, in any sufficiently small group of molecules belonging to a real body" [Maxwell 1878].

That is, statistical mechanics will not only derive thermodynamics, it will also correct it and extend it. That is especially true for nonequilibrium statistical mechanics as there we are necessarily dealing with atypical behavior from the point of view of the microcanonical ensemble. It therefore becomes both a major inspiration and application of probability theory, exactly in the way Lucio Russo has been enjoying and contributing to it.

Going to irreversible thermodynamics [de Groot and Mazur 1962], that is, the thermodynamics for irreversible phenomena, the main guiding principle that survives for the direction of currents is the positivity of the entropy production. We are for example considering an open macroscopic system which is being steadily frustrated by contacts with different equilibrium baths. Currents will be maintained, at least on the time scales where the environment is kept at the same intensive values (e.g., temperature). The directions of these currents can and will vary with different arrangements, but the entropy production $\Sigma$ is positive. We denote by $\Sigma=\sum_{\alpha} J_{\alpha} F_{\alpha}$ a sum over all possible types of channels of transport of the product of currents (or displacements) $J_{\alpha}$ and thermodynamic forces $F_{\alpha}$. For predicting the current directions, we just see what is compatible with $\Sigma \geq 0$, nothing more. In the linear regime, where currents are proportional to forces, $J_{\alpha}=\sum_{\gamma} L_{\alpha \gamma} F_{\gamma}$ with symmetric ${ }^{1}$ Onsager linear response coefficients $L_{\alpha \gamma}=L_{\gamma \alpha}$, and the positivity of $\Sigma$ is the positivity of the matrix $\left(L_{\alpha \gamma}\right)$. Here again, statistical mechanics will derive and extend that scheme, but now it should be nonequilibrium statistical mechanics. That is very much unfinished business and could certainly go beyond the linear regime around zero thermodynamic forces. In fact, nonequilibrium statistical mechanics is far behind the equilibrium version:

"My inclination is to postpone the study of the large-system limit: Since it is feasible to analyze the nonequilibrium properties of finite systems - as Gibbs did for their equilibrium properties - it seems a good idea to start there. That may not answer all questions, but it advances nonequilibrium statistical mechanics to the point equilibrium had reached after Gibbs" [Ruelle 2004].

In other words, a general theory of nonequilibrium phase transitions or of universality is still nonexistent, and even a systematic way of dealing with many-body

\footnotetext{
${ }^{1}$ We ignore here the Casimir correction that takes into account the parity under time-reversal of the physical quantity being transported.
} 


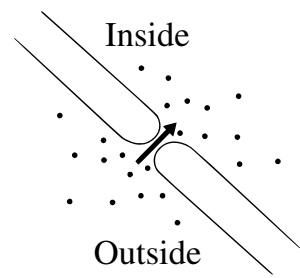

Figure 1. Example of a simple stationary current for which the direction is decided by the positivity of the entropy production.

effects is largely lacking. We certainly have no percolation or geometric picture of nonequilibrium collective phenomena, and remembering the crystal-clear and perfectly elegant contributions of Lucio to percolation theory and to mathematical statistical mechanics, we can only hope that the day will soon come when such a mathematical framework and geometric interpretation will also become available for nonequilibrium physics to match Lucio's standards.

In what follows, we are asking about what determines the direction of a nonequilibrium current. The main point will be that it is certainly not always the case that the current direction is decided by the positivity of the entropy production; nondissipative effects will be important and sometimes crucial. We refer to the pedagogical introduction [Maes 2015] on nondissipative aspects of nonequilibrium statistical mechanics. For the moment, it suffices to add that transition rates in a process also have time-symmetric parameters and, quite obviously, that we need to understand how they contribute to deciding the direction of the current.

\section{Traditional arguments}

2A. Phenomenology. The media inside and outside of a biological cell can be very different. These are connected via thin pores through which ions of various chemicals can be transported. Consider such a pore or channel in the membrane separating outside and inside; see Figure 1. Because of different concentrations at its ends, there will be a current through the pore. In fact, ions will be traveling from the region of higher chemical potential to the region of lower chemical potential. The same thing happens with many types of currents, whether the channel is connected to thermal, chemical or mechanical reservoirs. At the appropriate scale of time, the system is in steady nonequilibrium, not changing its macroscopic appearance. There is a constant production $\Sigma$ of entropy in the environment, which is positive,

$$
\Sigma=-\beta \mu_{1} J_{1}-\beta \mu_{2} J_{2}
$$

with $J_{i}$ the particle flux into the $i$-th reservoir at chemical potential $\mu_{i}$ and inverse temperature $\beta$. Stationarity (and bulk conservation of particles) implies $J_{1}+J_{2}=0$ 


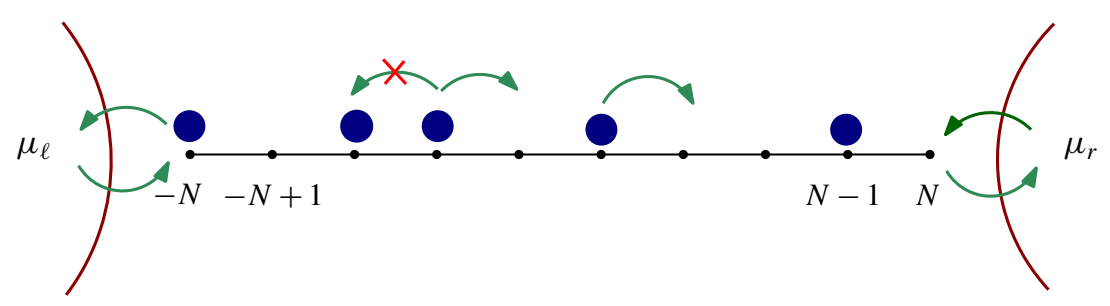

Figure 2. Stochastic lattice gas, symmetric in the bulk and governed by local interactions, driven by contact with particle reservoirs at different chemical potentials.

so that we can find the direction of the particle current $J_{1}$ by requiring

$$
\Sigma=\beta J_{1}\left(\mu_{2}-\mu_{1}\right) \geq 0 \quad \text { (second law). }
$$

By bulk conservation of particles, we still have $J_{1}=J$, the stationary particle current through the channel or pore from the second towards the first reservoir, and hence, $J \geq 0$ whenever $\mu_{2} \geq \mu_{1}$.

Similar scenarios can be written for thermal and mechanical baths that frustrate the system. Those are the typical cases where finding the direction of the current amounts to applying the second law in the form such that the stationary entropy production is positive.

While the previous case was treated rather phenomenologically, precise mathematical arguments can be provided for simple particle model systems following the same physics. Here comes an example.

2B. Stochastic lattice gas. We consider identical particles that can jump from site $i$ to the nearest neighbor site $j=i \pm 1$ on the finite linear chain $\Lambda_{N}=$ $\{-N,-N+1, \ldots, 0,1, \ldots, N-1, N\}$; see Figure 2. The endpoints $i= \pm N$ in $\Lambda_{N}$ are called the boundary of the system; the other sites are in the bulk. There is at most one particle per site $i$ so that a site $i$ can be vacant or occupied, and we write $\eta(i) \in\{0,1\}$ for the occupation at site $i \in \Lambda_{N}$. The state space is $K=\{0,1\}^{\Lambda_{N}}$ with elements $\eta, \eta^{\prime}, \xi, \ldots \in K$. The reasoning below is outlined in [Maes et al. 2009].

The energy function on $K$ is chosen as

$$
H(\eta)=-B \sum_{i=-N}^{N} \eta(i)-\kappa \sum_{i=-N}^{N-1} \eta(i) \eta(i+1),
$$

where $B$ and $\kappa$ are some real constants. The system is imagined in thermal contact with a very large heat bath at inverse temperature $\beta$ (Boltzmann's constant is set equal to 1). The energy change in that bath over the transition $\eta \rightarrow \eta^{\prime}$ gives a first contribution $\beta\left(H(\eta)-H\left(\eta^{\prime}\right)\right)$ to the change of entropy in the reservoir. Another 
important quantity here is the particle number

$$
\mathcal{N}_{[j, k]}(\eta)=\sum_{i=j}^{k} \eta(i)
$$

in the lattice interval $[j, k] \cap \Lambda_{N},-N \leq j \leq k \leq N$. The total number of particles inside the system is $\mathcal{N}=\mathcal{N}_{[-N, N] \text {. }}$

We now also imagine that the system is in contact with a particle reservoir at each of its boundary sites. There can be a birth or a death of a particle at these sites, which amounts to the entrance from and the exit to the corresponding reservoir of a particle. In that sense, we write $J_{\ell}=\Delta \mathcal{N}_{\ell}$ and $J_{r}=\Delta \mathcal{N}_{r}$ as the changes in particle number in the left and right particle reservoirs, respectively. The flow of particles in and out of the system can also contribute to the dissipated heat in the reservoir and hence to changes in entropy:

$$
S\left(\eta, \eta^{\prime}\right)=\beta\left[H(\eta)-H\left(\eta^{\prime}\right)\right]-\beta \mu_{\ell} \Delta \mathcal{N}_{\ell}\left(\eta, \eta^{\prime}\right)-\beta \mu_{r} \Delta \mathcal{N}_{r}\left(\eta, \eta^{\prime}\right)
$$

is the change of entropy in the environment for $\mu_{\ell}$ and $\mu_{r}$, the chemical potentials (up to some factor $\beta$ that we have ignored) of the left and right particle reservoirs, respectively. We will make mathematical sense of (2) entirely in terms of variables inside the system.

For the dynamics, we choose a continuous-time Markov process on $K$. Write the transformation

$$
\eta^{i, j}(k)= \begin{cases}\eta(k) & \text { if } k \neq i \text { and } k \neq j \\ \eta(i) & \text { if } k=j \\ \eta(j) & \text { if } k=i\end{cases}
$$

for the state obtained from $\eta$ after exchanging the occupation of the sites $i$ and $j$, only allowed for $j=i \pm 1$. The rate for that transition is taken to be

$$
C(i, j, \eta)=\exp \left[-\frac{\beta}{2}\left(H\left(\eta^{i, j}\right)-H(\eta)\right)\right], \quad|i-j|=1 .
$$

Similarly, the rate of birth and death for the transition $\eta \rightarrow \eta^{i}$ with

$$
\eta^{i}(k)= \begin{cases}1-\eta(k) & \text { if } k=i, \\ \eta(k) & \text { if } k \neq i,\end{cases}
$$

only occurring at sites $i=-N, N$, is

$$
C(i, \eta)=e^{-a_{i} \eta(i)} \exp \left[-\frac{\beta}{2}\left(H\left(\eta^{i}\right)-H(\eta)\right)\right] .
$$

The relevant parameters are the values $a_{-N}=\beta \mu_{\ell}$ and $a_{N}=\beta \mu_{r}$ representing the (different) chemical potentials of the two reservoirs at the outer edges. 
One observes from the definition (3) that

$$
\frac{C(i, j, \eta)}{C\left(i, j, \eta^{i, j}\right)}=\frac{\exp \left[-\beta H\left(\eta^{i, j}\right)\right]}{\exp [-\beta H(\eta)]}
$$

Furthermore, from (4),

$$
\frac{C(i, \eta)}{C\left(i, \eta^{i}\right)}=\frac{\exp \left[-a_{i} \eta(i)\right]}{\exp \left[-a_{i}(1-\eta(i))\right]} \frac{\exp \left[-\beta H\left(\eta^{i}\right)\right]}{\exp [-\beta H(\eta)]}, \quad i= \pm N .
$$

For $a_{-N}=a_{N}=a$, when the left and right particle reservoirs have equal concentration, then the system dynamics satisfies the condition of detailed balance: for all allowed transitions $\eta \rightarrow \eta^{\prime}$ and corresponding transition rates $W\left(\eta \rightarrow \eta^{\prime}\right)$,

$$
\frac{W\left(\eta \rightarrow \eta^{\prime}\right)}{W\left(\eta^{\prime} \rightarrow \eta\right)}=\frac{\mathbb{P}^{\beta, a}\left[\eta^{\prime}\right]}{\mathbb{P}^{\beta, a}[\eta]}
$$

for the grand-canonical equilibrium probabilities

$$
\mathbb{P}^{\beta, a}[\eta]=\frac{1}{\mathscr{L}} e^{a \sum \eta(i)} e^{-\beta H(\eta)}
$$

where $\mathscr{E}=\mathscr{L}(a, \beta, N)$ is a normalization factor. Thus, (8) is a reversible stationary measure when $a_{-N}=a_{N}=a$.

We now consider $a_{1} \neq a_{N}$ (different chemical potentials). At the left boundary of the system (see (6)),

$$
\frac{C(-N, \eta)}{C\left(-N, \eta^{-N}\right)}=e^{-\beta\left[H\left(\eta^{-N}\right)-H(\eta)\right]-a_{-N} J_{\ell}\left(\eta, \eta^{-N}\right)}
$$

where $J_{\ell}\left(\eta, \eta^{-N}\right)=1$ when the particle leaves the system via the site $-N$, i.e., $\eta(-N)=1$, and $J_{\ell}\left(\eta, \eta^{-N}\right)=-1$ when a new particle enters, i.e., $\eta(-N)=0$. Analogously, the current $J_{r}\left(\eta, \eta^{\prime}\right)=1$ when $\eta(N)=1$ and $\eta^{\prime}=\eta^{N}$ and $J_{\ell}\left(\eta, \eta^{\prime}\right)=$ -1 when $\eta(N)=0$ and $\eta^{\prime}=\eta^{N}$. The currents are zero otherwise.

As a consequence,

$$
\frac{W\left(\eta \rightarrow \eta^{\prime}\right)}{\left.W\left(\eta^{\prime} \rightarrow \eta\right)\right)}=e^{-\beta\left[H\left(\eta^{\prime}\right)-H(\eta)\right]-a_{-N} J_{\ell}\left(\eta, \eta^{\prime}\right)-a_{N} J_{r}\left(\eta, \eta^{\prime}\right)}
$$

where we see the change of entropy (2). In other words,

$$
\frac{W\left(\eta \rightarrow \eta^{\prime}\right)}{W\left(\eta^{\prime} \rightarrow \eta\right)}=e^{S\left(\eta, \eta^{\prime}\right)}
$$

(which is known as the condition of local detailed balance), and

$$
J_{\ell}\left(\eta, \eta^{\prime}\right)+J_{r}\left(\eta, \eta^{\prime}\right)=\mathcal{N}(\eta)-\mathcal{N}\left(\eta^{\prime}\right)
$$


or, with $a_{N}=a$ and $a_{-N}=a+\delta$,

$$
\frac{W\left(\eta \rightarrow \eta^{\prime}\right)}{W\left(\eta^{\prime} \rightarrow \eta\right)}=\frac{\mathbb{P}^{\beta, a}\left[\eta^{\prime}\right]}{\mathbb{P}^{\beta, a}[\eta]} e^{-\delta J_{\ell}\left(\eta, \eta^{\prime}\right)}
$$

with $\delta$ thus quantifying the amplitude of breaking of detailed balance.

As above, we define the bulk currents $J_{i}\left(\eta, \eta^{\prime}\right)$ to be +1 if in the transition $\eta \rightarrow \eta^{\prime}$ a particle moves over the bond $i \rightarrow i+1$ and equal to -1 if a particle moves $i \leftarrow i+1$. In fact, throughout, we confuse current with what is more like a time-integrated current or a change of particle number.

We have piecewise-constant paths $\omega$ over the time interval $[0, \tau]$, starting from some initial configuration $\eta_{0}$ after which it changes into $\eta_{t_{1}}, \eta_{t_{2}}, \ldots$ at random times $t_{1}, t_{2}, \ldots$ At the jump times, we take $\eta_{t_{k-1}}=\eta_{t_{k}^{-}}$and $\eta_{t_{k}}=\eta_{t_{k}^{+}}$for having right-continuous paths with left limits. The time-reversal transformation on pathspace $\Theta$ is defined via $(\Theta \omega)_{t}=\omega_{\tau-t}$, up to irrelevant modifications at the jump times making $\Theta \omega$ right-continuous again.

We consider a path $\omega=\left(\eta_{t}\right)_{t=0}^{\tau}$ and currents $J_{i}(\omega), i=-N, \ldots, N$, defined by

$$
J_{i}(\omega)=J_{i}\left(\eta_{0}, \eta_{t_{1}}\right)+J_{i}\left(\eta_{t_{1}}, \eta_{t_{2}}\right)+\cdots+J_{i}\left(\eta_{t_{n-1}}, \eta_{\tau}\right) .
$$

In particular, $J_{r}=J_{N}$ and for $i \leq k$

$$
\begin{aligned}
J_{i}(\omega)-J_{k}(\omega) & =\mathcal{N}_{[i+1, k]}\left(\eta_{\tau}\right)-\mathcal{N}_{[i+1, k]}\left(\eta_{0}\right), \\
J_{\ell}(\omega)+J_{-N}(\omega) & =\eta_{0}(-N)-\eta_{\tau}(-N) .
\end{aligned}
$$

Observe that the currents $J_{i}$ are extensive in the time $\tau$.

All of that is related to the process, be it transient or steady. We concentrate on the steady-state regime. It is easy to verify that we have here a unique stationary distribution $\rho$. That stationary distribution is only implicitly known and a solution of the (time-independent) master equation. Corresponding to $\rho$ is then a stationary process with distribution $\boldsymbol{P}_{\rho}$. If we look at expectations in the stationary process, we write $\langle\cdot\rangle_{\rho}$.

From the conservation laws (12) and (13),

$$
\left\langle J_{\ell}\right\rangle_{\rho}=-\left\langle J_{r}\right\rangle_{\rho}=-\left\langle J_{i}\right\rangle_{\rho}, \quad i \in \Lambda_{N} .
$$

Proposition 2.1. The direction of the current is from higher to lower chemical potential; i.e., assuming that $\delta \geq 0\left(\right.$ or $\left.a_{-N}=\mu_{\ell} \geq a_{N}=\mu_{r}\right)$, we have $\left\langle J_{i}\right\rangle_{\rho} \geq 0$.

Proof. The path density of $\boldsymbol{P}_{\rho}$ with respect to $\boldsymbol{P}_{\rho} \Theta$, both started in the stationary distribution $\rho$, is

$$
\frac{\mathrm{d} \boldsymbol{P}_{\rho}}{\mathrm{d} \boldsymbol{P}_{\rho} \Theta}(\omega)=\frac{\rho\left(\omega_{0}\right)}{\rho\left(\omega_{\tau}\right)} \exp \left[-\beta\left(H\left(\omega_{\tau}\right)-H\left(\omega_{0}\right)\right)+a \Delta \mathcal{N}-\delta J_{\ell}(\omega)\right]
$$


By normalization,

$$
\int \mathrm{d} \boldsymbol{P}_{\rho}(\omega) \frac{\mathrm{d} \boldsymbol{P}_{\rho} \Theta}{\mathrm{d} \boldsymbol{P}_{\rho}}(\omega)=1
$$

and hence, by concavity,

$$
\int \mathrm{d} \boldsymbol{P}_{\rho}(\omega) \log \frac{\mathrm{d} \boldsymbol{P}_{\rho} \Theta}{\mathrm{d} \boldsymbol{P}_{\rho}}(\omega) \leq 0 .
$$

But from (14) and by stationarity,

We conclude that

$$
0 \leq \int \mathrm{d} \boldsymbol{P}_{\rho} \log \frac{\mathrm{d} \boldsymbol{P}_{\rho}}{\mathrm{d} \boldsymbol{P}_{\rho} \Theta}(\omega)=-\delta\left\langle J_{\ell}\right\rangle_{\rho}=\delta\left\langle J_{i}\right\rangle_{\rho} .
$$

$$
\delta\left\langle J_{i}\right\rangle_{\rho} \geq 0
$$

which shows that the average direction of the particle current depends only on the sign of $\delta$.

Getting a strict inequality $\left\langle J_{i}\right\rangle_{\rho}>0$ is also possible for $\delta>0$; it suffices to see that there is a nonzero probability that the current $J_{i}$ as a function of the path $\omega$ is not identically equal to 0 even when $\omega_{0}=\omega_{\tau}$.

Looking back at the proof, we see that the main inequality has been the positivity (15) of the relative entropy between the forward and backward stationary processes. The latter coincides with the stationary entropy production, as is in fact visible from (11). Hence, the proof above, as in [Maes et al. 2009], is a nonperturbative statistical mechanical argument or the physical analogue for the phenomenology in Section 2A; there is nothing really new here.

\section{Problematic cases}

We collect a number of situations where either previous phenomenological or statistical mechanical arguments, based on the positivity of the entropy production, do not work. From a general perspective comparable to the so-called Curie principle, currents may appear whenever they are not forbidden by some symmetry. It is then not wholly surprising that we cannot always apply the same physical arguments. Yet the examples below are specifically relevant in the context of nonequilibrium physics, for which we may hope to develop some framework.

\section{A. Ratchet currents.}

3A1. Triangula. In [Van den Broeck et al. 2005], a number of versions of harddisk microscopic ratchets are introduced and studied with molecular dynamics and with some low-density expansions. A directed systematic motion appears when a temperature difference is applied to different units of a motor. One of the simple 


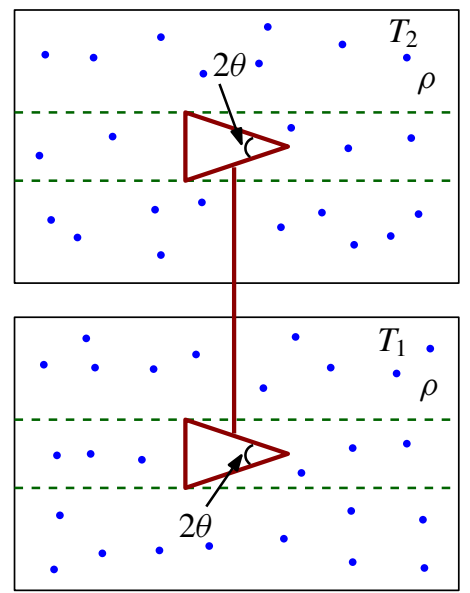

Figure 3. Triangula: the triangles can only move horizontally and are connected. They are in thermal baths at different temperatures.

examples is there called the Triangula: it is a motor consisting of two identical triangular units, each sitting in a gas (reservoir) consisting of hard disks whose centers collide elastically with the triangles; see Figure 3. The two triangles are rigidly connected along a rod, with their base parallel to it, and the whole motor is constrained to move along the horizontal direction without rotation or vertical displacement. When the temperatures in the two reservoirs are different, a systematic motion appears which turns out to be in the direction of the triangles' apices - to the right in Figure 3. The speed $V$ of the Triangula depends on the difference in temperatures $T_{1}$ and $T_{2}$ and on the apex angle $2 \theta$ and to some good approximation for low-density reservoirs is given by

$$
V=(1-\sin \theta) \frac{\sqrt{2 \pi k_{B} m}}{4 M}\left(T_{1}-T_{2}\right) \frac{\left(\sqrt{T_{1}}-\sqrt{T_{2}}\right)}{\left(\sqrt{T_{1}}+\sqrt{T_{2}}\right)^{2}}
$$

for $m$ the mass of the gas particle and $M$ the mass of the triangle (see (22) in [Van den Broeck et al. 2005] for the case of equal densities). If we write $T_{2}=$ $T(1+\varepsilon)$ and $T_{1}=T$, the leading order for that formula with $\varepsilon \downarrow 0$ becomes

$$
M V \simeq \frac{1-\sin \theta}{32} \sqrt{2 \pi k_{B} T m} \varepsilon^{2}
$$

and we see that the speed or current is second-order in the temperature difference. That is not attainable with linear response theory around equilibrium. The reason is that the translation current is orthogonal to the heat current (through the rod). Since we are thus in the regime of nonlinear response, that should already tell us that nondissipative features play a role [Basu et al. 2015]. As far as we know, nobody has a good heuristic or simple argument to explain that indeed $V>0$. Equation (16) 
connects the momentum of the Triangula with the thermal momentum of the gas, which appears rectified depending on the apex angle and is second-order between the temperatures, and is basically left without further explanation.

3A2. Parrondo game. The following is a paradoxical game invented by Juan Parrondo in 1996; see [Parrondo et al. 2000] for more explanations and references.

The state space is $K=\{1,2,3\}$, and the state at time $n$ is $x_{n}$. The Markov chain uses a different rule ( $A$ or $B$ ) at even and odd times $n$. Alternating, the following two games are played. Game $A$ is fair coin tossing: we simply move $x \rightarrow x \pm 1 \bmod 3$ with equal probability at even times. Game $B$ is played at odd times and with two biased coins, a good one and a bad one. In game $B$, the good coin is tossed when $x_{n} \in\{1,2\}$ and the bad coin is used each time when $x_{n}=3$. Winning takes $x_{n+1}=x_{n}+1$; losing at time $n$ means $x_{n+1}=x_{n}-1$, always modulo 3 . The transition probabilities are then

$$
\begin{array}{ll}
\operatorname{Prob}\left[x_{n+1}=x \pm 1 \mid x_{n}=x\right]=\frac{1}{2} & \text { when } n \text { is even, } \\
\operatorname{Prob}\left[x_{n+1}=x+1 \mid x_{n}=x\right]=\frac{3}{4} & \text { when } n \text { is odd and } x \neq 3, \\
\operatorname{Prob}\left[x_{n+1}=x+1 \mid x_{n}=x\right]=\frac{1}{10} & \text { when } n \text { is odd and } x=3 .
\end{array}
$$

Both games, when played separately at all times, are reversible. For example, for game $B$ (at all times), consider the cycle $3 \rightarrow 1 \rightarrow 2 \rightarrow 3$. Its stationary probability (always for game $B$ alone) is $\operatorname{Prob}[3 \rightarrow 1 \rightarrow 2 \rightarrow 3]=\rho(3) \times \frac{1}{10} \times \frac{3}{4} \times \frac{3}{4}=\frac{9}{160} \rho(3)$. For the reversed cycle, the probability $\operatorname{Prob}[3 \rightarrow 2 \rightarrow 1 \rightarrow 3]=\rho(3) \times \frac{9}{10} \times \frac{1}{4} \times \frac{1}{4}=$ $\frac{9}{160} \rho(3)$ is the same. The equilibrium distribution for game $B$ is then found to be $\rho(1)=\frac{2}{13}, \rho(2)=\frac{6}{13}$ and $\rho(3)=\frac{5}{13}$. Obviously then, there is no current when playing game $B$ and clearly the same is trivially verified for game $A$ when tossing with the fair coin. Yet, and here is the paradox, when periodically playing game $B$ after game $A$, a current arises.

As in the previous case of the Triangula, the very fact that a current arises is again not so strange, but the question is what really decides its direction. We will show how to solve that question for a continuous-time version at low temperature in Section 4.

3B. Multiple cycles. It is not uncommon in nonequilibrium to have multiple cycles in state space along which the dynamics can proceed. We give here two examples of one-dimensional random walks, visualized in Figures 4-6. Look first at Figure 4 , where we denote the states by $\{0, u, 1\}$ in an elementary triangle. For horizontal motion, there are two "channels" to move to the right, $0 \rightarrow u \rightarrow 1$ and $0 \rightarrow 1$, and two "channels", $0 \rightarrow u \rightarrow-1$ and $0 \rightarrow-1$, to move to the left. Going right, the system prefers the "channel" $0 \rightarrow u \rightarrow 1$, and for going left, the system 




Figure 4. Necklace of three-state cycles with rotational current inducing a horizontal current.

prefers the channel $0 \rightarrow-1$ as we take the transition rates

$$
\begin{array}{lll}
k(1,0)=\varphi e^{\varepsilon / 2}, & k(0, u)=e^{\varepsilon / 2}, & k(u, 1)=e^{\varepsilon / 4}, \\
k(0,1)=\varphi e^{-\varepsilon / 2}, & k(u, 0)=1, & k(1, u)=e^{-\varepsilon / 4}
\end{array}
$$

for parameters $\varphi, \varepsilon>0$. That is periodically repeated to obtain a random walk on the line. We are interested in the physical "translational" current towards the right, that is,

$$
J=\rho(0)\left[e^{\varepsilon / 2}+\varphi e^{-\varepsilon / 2}\right]-\left[\rho(u)+\rho(0) \varphi e^{\varepsilon / 2}\right]
$$

where we restrict ourselves to translation-invariant stationary occupations satisfying the normalization $\rho(0)+\rho(u)=1$ and

$$
\rho(0)\left[e^{\varepsilon / 2}+e^{-\varepsilon / 4}\right]=\rho(u)\left[1+e^{\varepsilon / 4}\right] .
$$

We claim that entropy production decides the orientation of the rotational current within each triangle but not the direction of the induced translational current $J$. The $\varepsilon$ in (18) decides the direction of the rotational current and is responsible for the breaking of detailed balance. It stands for an entropy flux (per $k_{B}$ ). For example, the trajectory $0 \rightarrow u \rightarrow 1$ (taking the walker one step to the right) expends an entropy flux $\varepsilon$ (e.g., in the sense of [Maes et al. 2000]), as seen from the calculation

$$
\frac{k(0, u) k(u, 1)}{k(u, 0) k(1, u)}=\frac{e^{\varepsilon / 2} e^{\varepsilon / 4}}{1 \cdot e^{-\varepsilon / 4}}=e^{\varepsilon},
$$

but so does the step $0 \rightarrow-1$ exactly: taking the walker one step to the left,

$$
\frac{k(1,0)}{k(0,1)}=e^{\varepsilon} .
$$

In all, there is no entropic preference to go right or left. In other words, the effective bias is also decided by the parameter $\varphi$. We see that in Figure 5.

The current $J$ is plotted in Figure 5, left, as a function of $\varepsilon$ for two different choices of $\varphi$. Fixing say $\varepsilon=6$, we see a positive current for $\varphi=0.75$ and a negative current for $\varphi=0.90$. In other words, the direction of the current is not simply decided. The current diverges like $(1-\varphi) \exp [\varepsilon / 4]$ for $\varphi \neq 1$ as $\varepsilon \uparrow \infty$. If $\varphi$ is large, the current is to the left, and if $\varphi$ is small, the current becomes positive. For $\varphi>\frac{1}{2}$, there is a sign-reversal in the current as a function of the entropy flux $\varepsilon$. 


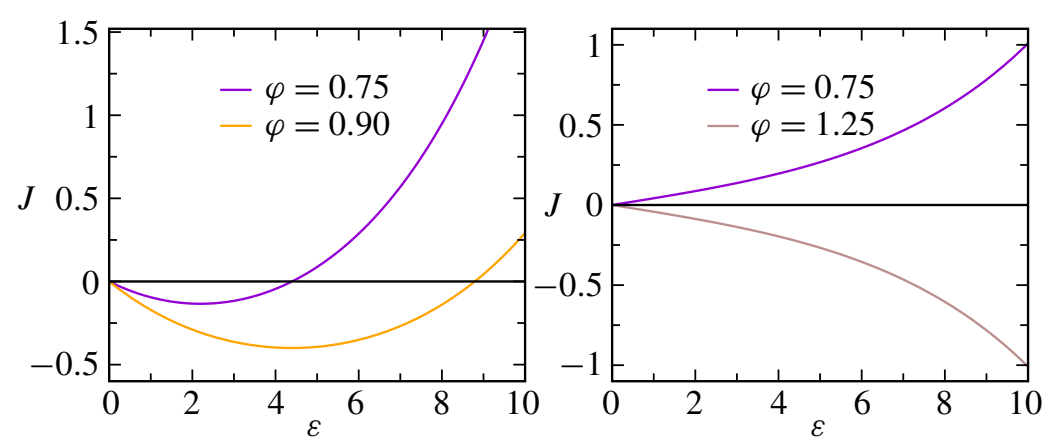

Figure 5. The horizontal current towards the right as a function of $\varepsilon$ corresponding to (left) Figure 4 for $\varphi=0.75$ (upper) and $\varphi=0.90$ (lower curve) and to (right) Figure 6 for $\varphi=0.75$ (upper) and $\varphi=1.25$ (lower curve).

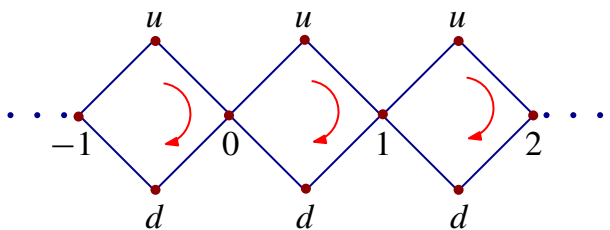

Figure 6. Four-state necklace with top versus bottom symmetry for $\varphi=1$.

We can add more symmetry in the construction by considering, minimally, a four-state Markov process as an elementary unit. Then we look at Figure 6, denoting the states by $\{0, u, 1, d\}$ (where $u$ stands for "up" and $d$ stands for "down"), and the transition rates are

$$
\begin{array}{llll}
k(1, d)=\varphi e^{\varepsilon / 4}, & k(d, 0)=\varphi e^{\varepsilon / 4}, & k(0, u)=e^{\varepsilon / 4}, & k(u, 1)=e^{\varepsilon / 4}, \\
k(d, 1)=\varphi e^{-\varepsilon / 4}, & k(0, d)=\varphi e^{-\varepsilon / 4}, & k(u, 0)=e^{-\varepsilon / 4}, & k(1, u)=e^{-\varepsilon / 4} .
\end{array}
$$

We again have two elementary paths in the opposite direction, which are now $R_{1}$ : $0 \rightarrow u \rightarrow 1$ and $R_{2}: 0 \rightarrow d \rightarrow-1$, for which the entropy fluxes are both equal to $\varepsilon$. (Of course, $R_{2}$ can be identified with the path $1 \rightarrow d \rightarrow 0$.) It will again be the "reactivity" $\varphi>0$ that also decides the direction of the current; see Figure 5, right. Or what starts out as a time-symmetric parameter turns out to give rise to time-asymmetry.

The above scenario has natural realizations, e.g., in the motion of some molecular motors like Myosin V studied in [Maes and O'Kelly de Galway 2015]. The $\varphi$ then corresponds to the activity of the leading head. Because it is lower than that of the trailing head, the motor moves forward. The relation between dynamical activity or "happy feet" (of Paulo Conte's song) and the direction of current has been anticipated in the Inferno (Canto I) of the Divine Comedy, where Dante writes, 

"E come quei che con lena affannata,
uscito fuor del pelago a la riva, si volge a l'acqua perigliosa e guata,
così l'animo mio, ch'ancor fuggiva, si volse a retro a rimirar lo passo che non lasciò già mai persona viva.
Poi ch'èi posato un poco il corpo lasso, ripresi via per la piaggia diserta, sì che 'l piè fermo sempre era 'l più basso" [Dante 1787, p. 2].

In Figure 6, one should imagine the top corresponding to lifting the trailing foot and the bottom to lifting the leading foot; $\varphi>1$ corresponds to a more "active" trailing foot which easily moves you forward. In the case of Dante leaving the valley and climbing the mountain, as the firm or more stable foot was always the lower $(\varphi<1)$, it becomes very difficult to go forward and not to retreat.

3C. Nonequilibrium internal degrees of freedom. We are used to thinking of internal degrees of freedom as an equilibrium reservoir. When a ball bounces off the ground, it slightly deforms and warms up, indicating a restitution coefficient which is less than 1 [Maes and Tasaki 2007]; the entropy gets dissipated in these many internal degrees of freedom. But what if the ball is "alive" or "active", or to put it in less suggestive language, what if the internal degrees of freedom are in steady nonequilibrium? Can that not produce extra interesting effects? The problematic case of the Triangula in Section 3A1 can be seen as an example. The two triangles connected by the vertical rod shown in Figure 3 make one extended object which internally is subject to heat conduction (vertical energy current from higher to lower temperature).

Look now at Figure 7 for greater simplification. Our object has position $q \in S^{1}$ on the ring suspended in a thermal bath at inverse temperature $\beta$. For its dynamics, we assume the overdamped Langevin dynamics

$$
\gamma \dot{q}=-\frac{\partial}{\partial q} E(x, q)+\sqrt{\frac{2 \gamma}{\beta}} \xi_{t}
$$

in the usual physics notation with $\xi_{t}$ standard white noise, $\gamma$ the damping coefficient and $E(x, q)$ some interaction potential with an "internal" degree of freedom, here a four-state Markov process with $x=1,2,3,4$. We assume that the $x$ relaxes fast to stationarity compared to the walker where the time scale is set by $\gamma$, and we take transition rates

$$
k^{q}\left(x, x^{\prime}\right)=e^{-(\beta / 2)\left[E\left(x^{\prime}, q\right)-E(x, q)\right]} \varphi\left(x, x^{\prime}\right) e^{s\left(x, x^{\prime}\right) / 2} .
$$




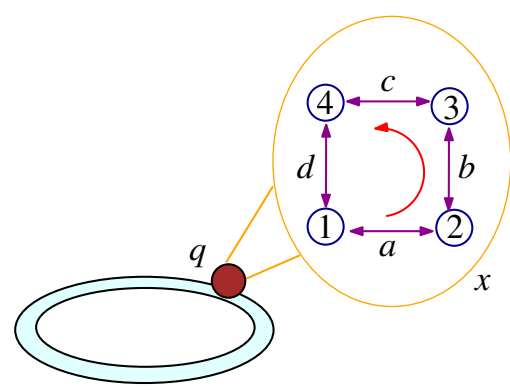

Figure 7. Walker (probe or colloid) on a ring with position $q$ with rotating stomach $x \in\{1,2,3,4\}$. The joint dynamics is specified in (19)-(20). The colloid's position is the slow degree of freedom.

The driving or nonequilibrium sits in $s(1,2)=s(2,3)=s(3,4)=s(4,1)=\beta \varepsilon$ and the symmetric $\varphi\left(x, x^{\prime}\right)$ are $\varphi(1,2)=a, \varphi(2,3)=b, \varphi(3,4)=c$ and $\varphi(4,1)=d$; see the "stomach" in Figure 7. Under the hypothesis of infinite time-scale separation, the colloid is subject to the mean force

$$
f(q)=-\sum_{x} \rho_{q}(x) \frac{\partial}{\partial q} E(x, q)
$$

which can be calculated exactly from the stationary distribution $\rho_{q}(x)$ for the internal degree of freedom $x$. When the rotational part of the force $f_{\text {rot }}=\oint f(q) \mathrm{d} q$ is nonzero, then the colloid will start moving around the circle. In fact, the steady current $J$, as plotted in Figure 8, is essentially just given by it. Obviously, there are many parameters: the form of the potential $E(x, q)$ but also the coefficients $a$, $b, c$ and $d$. We ask here what determines the sign of that rotational force, which of course determines the direction of the current of the walker.

We observe here that we can get a sign-reversal of the current by solely varying the kinetic factors $a, b, c$ and $d$. More specifically, we consider the energy function $E(x, q)$ for $q \in[-1,1], E(2, q)=E(4, q)=0$,

$$
E(1, q)=\left\{\begin{array}{ll}
\frac{4}{7}(1+q) & \text { for } q \leq \frac{3}{4}, \\
4(1-q) & \text { for } q \geq \frac{3}{4}
\end{array} \quad \text { and } \quad E(3, q)= \begin{cases}4(1+q) & \text { for } q \leq-\frac{3}{4} \\
\frac{4}{7}(1-q) & \text { for } q \geq-\frac{3}{4}\end{cases}\right.
$$

In Figure 8, left, we see the rotational current $J=f_{\text {rot }}$ as a function of $c$ for $a=b=d=1$ (first negative then positive) and as a function of $a$ for $b=c=$ $d=1$ (first positive then negative), both at driving $\varepsilon=5$ and $\beta=1$. The same is represented in the right panel but now as a function of the driving $\varepsilon$. We clearly get information about the time-symmetric part in the transition rates (20) from coupling that process $x_{t}$ to the position $q_{t}$ in (19) of the walker and measuring its induced current. 

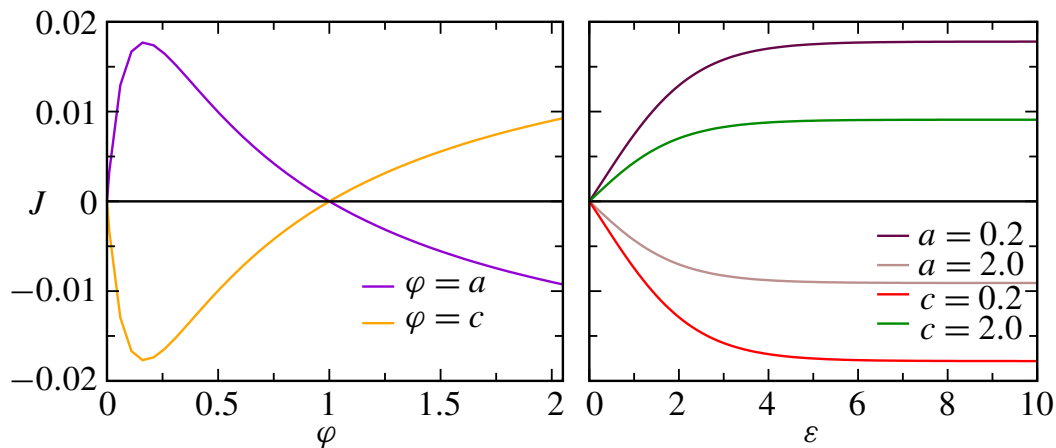

Figure 8. The rotational current $J=f_{\text {rot }}=\oint f(q) \mathrm{d} q$ of the colloid as it depends on the reactivities $\varphi$ and the driving $\varepsilon$ of the internal nonequilibrium in Figure 7; see (20). Left: the direction of the current can change as a function of $a, b, c$ and $d$; we see the current at $\varepsilon=5$ and $\beta=1$ as a function of $\varphi=a$ and as a function of $\varphi=c$, while the other reactivities equal 1 when not specified. Right: the current as a function of the driving $\varepsilon$ for various choices of $\varphi\left(x, x^{\prime}\right)$. From low to high, the curves correspond to $c=0.2$, $a=2.0, c=2.0$ and $a=0.2$ again with all other reactivities fixed to the value 1 when not specified.

3D. Wrong direction! The response to an external field can be negative. It is then the case that by pushing harder the particle gets slower. It could even happen that by pushing in one direction the particle moves in the opposite direction making negative absolute conductivity. In [Cleuren and Van den Broeck 2002], one uses memory to achieve that result, but one also gets it from considering the Markov models of Section 3B.

Consider again the setup of Figure 4 and the resulting Figure 5, left, for the horizontal current in the positive direction. There are possible stalling points $\left(\varepsilon^{*}, \varphi^{*}\right)$ where that current vanishes. Taking these values or, more generally, fixing arbitrary $(\varepsilon, \varphi)$, we perturb the rates $(18)$ as

$$
\begin{array}{lll}
k_{E}(1,0)=[\varphi+E] e^{\varepsilon / 2}, & k_{E}(0, u)=e^{[\varepsilon+E] / 2}, & k_{E}(u, 1)=e^{[\varepsilon+E] / 4}, \\
k_{E}(0,1)=[\varphi+E] e^{-\varepsilon / 2}, & k_{E}(u, 0)=1, & k_{E}(1, u)=e^{-\varepsilon / 4},
\end{array}
$$

pushing a bit harder with $E>0$ in the upper channel (only) and also changing the time-symmetric coefficient $\varphi \rightarrow \varphi+E$. We get a new value of the horizontal current $J_{E}$, and we can ask how it changes, that is, find the conductivity

$$
\sigma=\left.\frac{\mathrm{d} J_{E}}{\mathrm{~d} E}\right|_{E=0} .
$$




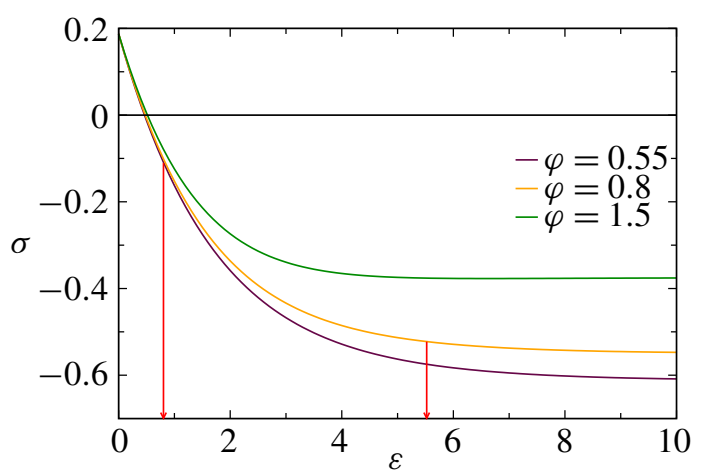

Figure 9. The conductivity $\sigma$ (22), rescaled by a factor of $\exp [-\varepsilon / 4]$, as a function of $\varepsilon$ for $\varphi=0.55$ (lowest curve) having its stalling point at $\varepsilon^{*} \simeq 0.80$, for $\varphi=0.8$ with stalling point at $\varepsilon^{*} \simeq 5.54$ and $\varphi=1.5$ (upper curve). There is negative conductivity $\sigma<0$, including at stalling points where the pushing makes the walker go back instead of forward.

We see in Figure 9 that $\sigma$ gets negative for large-enough values of $\varepsilon$, including at stalling values. Thus, there, the current actually goes backward while pushing forward.

\section{Low temperature analysis}

We consider here a continuous-time version of the Parrondo game of Section 3A2 with random flipping between a flat potential and a nontrivial energy landscape. It gives an approach to the problems of Sections 3A2-3B by considering lowtemperature asymptotics. In particular, we use the Freidlin-Wentzell theory of [Maes et al. 2014] to obtain an expression for the low-temperature ratchet current. Its direction is not determined by entropic considerations (only) but involves the reactivities.

Look at Figure 10. States of a continuous-time Markov process are on two rings, each having $N>2$ of states, denoted by $x=(i, n)$ where $i \in\{1=N+1,2, \ldots, N\}$ and $n=0,1$.

On the outer ring $(n=0)$, energies $E_{1}<\cdots<E_{N}$ are associated to the states and transition rates are thermal:

$$
k((i, 0),(i+1,0))=e^{(\beta / 2)\left(E_{i}-E_{i+1}\right)}, \quad k((i+1,0),(i, 0))=e^{(\beta / 2)\left(E_{i+1}-E_{i}\right)}
$$

for inverse temperature $\beta$. The inner ring $(n=1)$ corresponds to a walker in a flat potential landscape so that

$$
k((i, 1),(i+1,1))=k((i+1,1),(i, 1))=1 .
$$




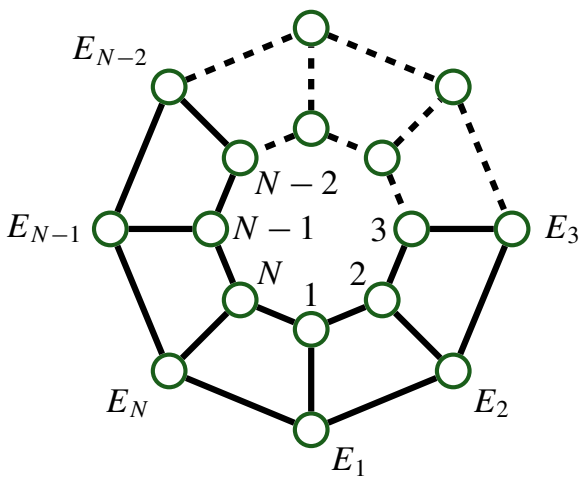

Figure 10. Continuous-time Parrondo game.

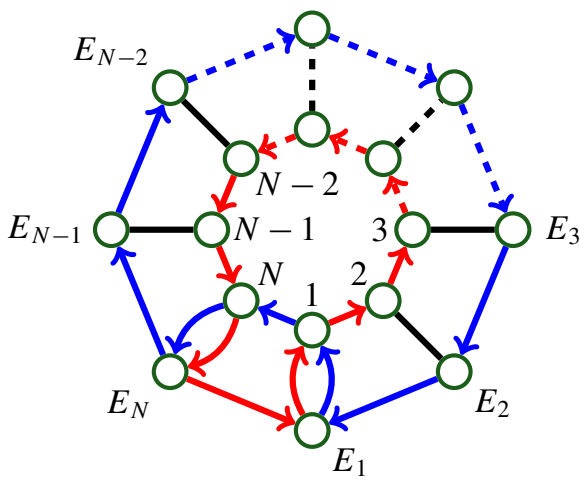

Figure 11. Trajectories $\omega_{1}$ (clockwise, blue) and $\omega_{2}$ (counterclockwise, red) with the same entropy flux yet in opposite directions.

The random flipping between the two potentials is realized by moves between the rings $n=0,1$, at transition rates $k((i, n),(i, 1-n))=a$ for some $a>0$. There is no explicit driving except that for $a=0$ there is detailed balance of course and for very strong coupling $a \gg 1$ the model is effectively running on a single ring. In the limit $a \uparrow \infty$, there is again detailed balance with inverse temperature $\beta / 2$.

The question for the nonequilibrium situation is in what sense the walker will typically move: either clockwise of counterclockwise. Again, the direction of that current, which we now call the ratchet current, is not decided by the positivity of the entropy production. Consider for example Figure 11, where two trajectories $\omega_{1}=((N, 0),(N-1,0), \ldots,(1,0),(1,1),(N, 1),(N, 0))$ and $\omega_{2}=$ $((N, 0),(1,0),(1,1),(2,1), \ldots,(N, 1),(N, 0))$ are depicted that wind in opposite directions yet whose entropy fluxes are exactly identical, equal to $s\left(\omega_{1}\right)=$ $s\left(\omega_{2}\right)=\beta\left(E_{N}-E_{1}\right)>0$. 
The stationary ratchet current $J_{R}$ in the clockwise direction is

$$
J_{R}=j((i+1,0),(i, 0))+j((i+1,1),(i, 1))
$$

with $j(x, y)=k(x, y) \rho(x)-k(y, x) \rho(y)$ where $\rho$ is the stationary probability law for the Markov dynamics (23)-(24). $J_{R}$ is the current over both rings together, and of course, that current also depends on the size $N$, on the energies and on temperature. We will look at the case $a=1$ but at low temperatures so that the transitions $(i, 0) \rightarrow(i+1,0)$ are exponentially damped. It simplifies the structure of the stationary distribution $\rho$; see [Maes et al. 2014], where a type of FreidlinWentzell analysis is applied to find its low-temperature asymptotics. The following combines proofs in [Maes et al. 2014] and in [Louis 2015] to show that the ratchet current is clockwise and saturates:

Proposition 4.1. $J_{R}=0$ for $N=3, J_{R}>0$ for all $N>3$ and $\lim _{N \uparrow \infty} J_{R}=\frac{1}{2}-\frac{1}{\sqrt{5}}$.

Proof. Consider the set $\mathscr{D}:=\{(1,0),(i, 1): i=1, \ldots, N\}$, and let $M(x)$ be the number of in-spanning trees in the digraph obtained from Figure 10 by keeping only the oriented bonds $(v, w)$ where $w$ is one of the most likely successors of $v$. From [Maes et al. 2014], we learn that at low temperatures $\rho(x) \propto|M(x)|$ for $x \in \mathscr{D}$ and $\rho(y) \simeq|M(y)| e^{\beta \Gamma(y)} / \mathscr{E}$, with some $\Gamma(y)<0$ for $y \notin \mathscr{D}$. By the matrix-tree theorem (see, e.g., [Tutte 1984]), we need the Laplacian matrix $L$ on the digraph $K^{D}$, and we erase the row and the column corresponding to vertex $x$ to obtain the matrix $L_{x}$. Then

$$
|M(x)|=\operatorname{det} L_{x} .
$$

The Laplacian of the digraph $K^{D}$ has a rather simple structure:




The state for which the number of in-trees becomes maximal is $(1,0)$ : there are more combinations to form an in-tree to $(1,0)$ than to any other state $(i, 1)$ on the inner ring.

To compute the ratchet current, we take $x=(1,1)$ with $\rho(1,1) \simeq A((1,1)) / \mathscr{E}$. Then

$$
j((2,1),(1,1)) \simeq \frac{1}{\mathscr{L}}(A((2,1))-A((1,1))) .
$$

Moreover,

$$
j((2,0),(1,0)) \simeq \frac{A((2,0))}{\mathscr{L}} .
$$

As a consequence,

$$
J_{R} \simeq \frac{1}{\mathscr{L}}\left(\operatorname{det} L_{(2,1)}+\operatorname{det} L_{(2,0)}-\operatorname{det} L_{(1,1)}\right) .
$$

Furthermore, by inspecting the Laplacian $L$, one finds that

- $\operatorname{det} L_{(2,0)}=2 \operatorname{det} B_{N-1}-3 \operatorname{det} B_{N-2}-3$,

- $\operatorname{det} L_{(1,1)}=\operatorname{det} B_{N-1}$ and

- $\operatorname{det} L_{(2,1)}=\operatorname{det} B_{N-2}+1$

with

$$
B_{N}=\left(\begin{array}{rrrr}
3 & -1 & & \\
-1 & 3 & \ddots & \\
& \ddots & \ddots & -1 \\
& & -1 & 3
\end{array}\right) \text {. }
$$

$B_{N}$ satisfies the recursion relation $\operatorname{det} B_{N}=3 \operatorname{det} B_{N-1}-\operatorname{det} B_{N-2}$, where det $B_{2}=8$ and $\operatorname{det} B_{1}=3$. Hence, by solving the recurrence, we get

$$
\operatorname{det} B_{N}=\frac{5-3 \sqrt{5}}{10}\left(\frac{3-\sqrt{5}}{2}\right)^{N}+\frac{5+3 \sqrt{5}}{10}\left(\frac{3+\sqrt{5}}{2}\right)^{N}
$$

to be used in

$$
J_{R} \simeq \frac{\operatorname{det} B_{N-1}-2 \operatorname{det} B_{N-2}-2}{\mathscr{L}}
$$

which already proves that $J_{R}>0$ for all $N \geq 4$ and $J_{R}=0$ when $N=3$; the direction is clockwise. For the $N$-asymptotics, we also need the normalization $\mathscr{L}$. In fact, $\mathscr{L} \simeq \sum_{x \in \mathscr{D}}|M(x)|=\sum_{x \in \mathscr{D}} \operatorname{det} L_{x}$. In [Louis 2015], it is shown that

$$
\mathscr{L} \simeq 2\left(\frac{3-\sqrt{5}}{2}\right)^{N}+2\left(\frac{3-\sqrt{5}}{2}\right)^{N}-4,
$$

which concludes the proof by a simple computation. 
Note that adding particles and interactions we can get direction-reversal of currents as we had in the previous Sections 3B-3C. An experimentally accessible example is described in [de Souza Silva et al. 2006]. That constitutes a big challenge in the discussion of the direction of currents that has not been touched upon here: how density and interactions can modify it.

\section{Conclusion}

To discover what decides the direction of a current under steady nonequilibrium conditions is a major challenge of statistical mechanics. In the present paper, we have seen that many effects are possible, not least from the variation of timesymmetric parameters in transition rates defining the process. That dependence on nondissipative aspects thus provides a method to obtain kinetic parameters from measuring the direction of the current.

\section{Acknowledgment}

I thank Urna Basu for many discussions and help.

\section{References}

[Basu et al. 2015] U. Basu, M. Krüger, A. Lazarescu, and C. Maes, "Frenetic aspects of second order response", Phys. Chem. Chem. Phys. 17 (2015), 6653-6666.

[Van den Broeck et al. 2005] C. Van den Broeck, P. Meurs, and R. Kawai, "From Maxwell demon to Brownian motor", New J. Phys. 7 (2005), 10.

[Cleuren and Van den Broeck 2002] B. Cleuren and C. Van den Broeck, "Random walks with absolute negative mobility”, Phys. Rev. E 65 (2002), 030101(R).

[Dante 1787] Dante, Inferno, Paris, C. A. I. Jacob, 1787.

[de Groot and Mazur 1962] S. R. de Groot and P. Mazur, Non-equilibrium thermodynamics, NorthHolland, Amsterdam, 1962.

[Louis 2015] J. Louis, "Low temperature ratchet current”, preprint, 2015. arXiv 1508.01189v1

[Maes 2015] C. Maes, “Non-dissipative effects in nonequilibrium systems”, lecture notes, 2015. arXiv 1603.05147v1

[Maes and O'Kelly de Galway 2015] C. Maes and W. O'Kelly de Galway, "On the kinetics that moves Myosin V”, Phys. A 436 (2015), 678-685.

[Maes and Tasaki 2007] C. Maes and H. Tasaki, "Second law of thermodynamics for macroscopic mechanics coupled to thermodynamic degrees of freedom", Lett. Math. Phys. 79:3 (2007), 251-261.

[Maes et al. 2000] C. Maes, F. Redig, and A. Van Moffaert, "On the definition of entropy production, via examples", J. Math. Phys. 41:3 (2000), 1528-1554.

[Maes et al. 2009] C. Maes, K. Netočný, and B. Shergelashvili, "A selection of nonequilibrium issues", pp. 247-306 in Methods of contemporary mathematical statistical physics, edited by R. Kotecký, Lecture Notes in Math. 1970, Springer, Berlin, 2009.

[Maes et al. 2014] C. Maes, K. Netočný, and W. O’Kelly de Galway, "Low temperature behavior of nonequilibrium multilevel systems", J. Phys. A Math. Theor. 47:3 (2014), 035002. 
[Maxwell 1878] J. C. Maxwell, “Tait's 'Thermodynamics', II”, Nature 17:432 (1878), 278-280.

[Parrondo et al. 2000] J. M. R. Parrondo, G. P. Harmer, and D. Abbott, "New paradoxical games based on Brownian ratchets", Phys. Rev. Lett. 85:24 (2000), 5226-5229.

[Ruelle 2004] D. Ruelle, "Conversations on nonequilibrium physics with an extraterrestrial", Phys. Today 57:5 (2004), 48-53.

[de Souza Silva et al. 2006] C. C. de Souza Silva, J. Van de Vondel, M. Morelle, and V. V. Moshchalkov, "Controlled multiple reversals of a ratchet effect", Nature 440:7084 (2006), 651-654.

[Tutte 1984] W. T. Tutte, Graph theory, Encyclopedia of Mathematics and its Applications 21, Addison-Wesley, Menlo Park, CA, 1984.

Received 1 Apr 2016. Revised 19 Aug 2016. Accepted 26 Sep 2016.

Christian MAES: christian.maes@fys.kuleuven.be

Instituut voor Theoretische Fysica, Katholieke Universiteit Leuven, Celestijnenlaan 200D, 3001 Leuven, Belgium 
EDITORIAL BOARD

ANTONIO CARCATERRA

ERIC A. CARLEN

FRANCESCO DELL'ISOLA

RAFFAELE ESPOSITO

ALBERT FANNJIANG

Gilles A. FranCFORT

Pierangelo MARCATI

JEAN-JACQUES MARIGO

PETER A. MARKOWICH

MARTIN OSTOJA-STARZEWSKI

PIERRE SEPPECHER

DAVID J. STEIGMANN

PAUl STEINMANN

PierRe M. SuQueT

MANAGING EDITORS

MICOL AMAR

CORRADO LATTANZIO

ANGELA MADEO

MARTIN OSTOJA-STARZEWSKI

ADVISORY BOARD

ADNAN AKAY

Holm AltenBaCH

MICOL AMAR

HARM ASKES

TEODOR ATANACKOVIĆ

VICTOR BERDICHEVSKY

GUY BOUCHITTÉ

ANDREA BRAIDES

ROBERTO CAMASSA

MAURO CARFORE

ERIC DARVE

FELIX DARVE

ANNA DE MASI

GianPiEtro DEL Piero

EMMANUELE Di BENEDETTO

BERNOLD FIEDLER

IRENE M. GAMBA

DAVID Y. GAO

SERGEY GAVRILYUK

TIMOTHY J. HEALEY

DOMINIQUE JEULIN

ROGER E. KHAYAT

CORRADO LATTANZIO

ROBERT P. LIPTON

ANGELO LUONGO

ANGELA MADEO

JUAN J. MANFREDI

CARLO MARCHIORO

GÉRARD A. MAUGIN

ROBERTO NATALINI PATRIZIO NEFF

ANDREY PIATNITSKI

ERRICO PRESUTTI

MARIO PULVIRENTI

LUCIO RUSSO

Miguel A. F. SANJUAN

PATRICK SElVADURAI

ALEXANDER P. SEYRANIAN

MIROSLAV ŠILHAVÝ

GUIDO SWEERS

ANTOINETTE TORDESILLAS

LEV TRUSKINOVSKY

JUAN J. L. VELÁZQUEZ VINCENZO VESPRI ANGELO VULPIANI msp.org/memocs

Università di Roma "La Sapienza", Italia

Rutgers University, USA

(CO-CHAIR) Università di Roma "La Sapienza", Italia

(TREASURER) Università dell'Aquila, Italia

University of California at Davis, USA

(CO-CHAIR) Université Paris-Nord, France

Università dell'Aquila, Italy

École Polytechnique, France

DAMTP Cambridge, UK, and University of Vienna, Austria

(CHAIR MANAGING EDITOR) Univ. of Illinois at Urbana-Champaign, USA

Université du Sud Toulon-Var, France

University of California at Berkeley, USA

Universität Erlangen-Nürnberg, Germany

LMA CNRS Marseille, France

Università di Roma "La Sapienza", Italia

Università dell'Aquila, Italy

Université de Lyon-INSA (Institut National des Sciences Appliquées), France

(CHAIR MANAGING EDITOR) Univ. of Illinois at Urbana-Champaign, USA

Carnegie Mellon University, USA, and Bilkent University, Turkey

Otto-von-Guericke-Universität Magdeburg, Germany

Università di Roma "La Sapienza", Italia

University of Sheffield, UK

University of Novi Sad, Serbia

Wayne State University, USA

Université du Sud Toulon-Var, France

Università di Roma Tor Vergata, Italia

University of North Carolina at Chapel Hill, USA

Università di Pavia, Italia

Stanford University, USA

Institut Polytechnique de Grenoble, France

Università dell'Aquila, Italia

Università di Ferrara and International Research Center MEMOCS, Italia

Vanderbilt University, USA

Freie Universität Berlin, Germany

University of Texas at Austin, USA

Federation University and Australian National University, Australia

Université Aix-Marseille, France

Cornell University, USA

École des Mines, France

University of Western Ontario, Canada

Università dell' Aquila, Italy

Louisiana State University, USA

Università dell'Aquila, Italia

Université de Lyon-INSA (Institut National des Sciences Appliquées), France University of Pittsburgh, USA

Università di Roma "La Sapienza”, Italia

Université Paris VI, France

Istituto per le Applicazioni del Calcolo "M. Picone", Italy

Universität Duisburg-Essen, Germany

Narvik University College, Norway, Russia

Università di Roma Tor Vergata, Italy

Università di Roma "La Sapienza”, Italia

Università di Roma “Tor Vergata”, Italia

Universidad Rey Juan Carlos, Madrid, Spain

McGill University, Canada

Moscow State Lomonosov University, Russia

Academy of Sciences of the Czech Republic

Universität zu Köln, Germany

University of Melbourne, Australia

École Polytechnique, France

Bonn University, Germany

Università di Firenze, Italia

Università di Roma La Sapienza, Italia

MEMOCS (ISSN 2325-3444 electronic, 2326-7186 printed) is a journal of the International Research Center for the Mathematics and Mechanics of Complex Systems at the Università dell'Aquila, Italy.

Cover image: "Tangle” by $\odot$ John Horigan; produced using the Context Free program (contextfreeart.org).

PUBLISHED BY

7 mathematical sciences publishers

nonprofit scientific publishing

http://msp.org/

(C) 2016 Mathematical Sciences Publishers 
Special issue in honor of

Lucio Russo

Lucio Russo: A multifaceted life

Raffaele Esposito and Francesco dell'Isola

The work of Lucio Russo on percolation Geoffrey R. Grimmett

"Mathematics" and "physics" in the science of harmonics Stefano Isola

From quantum to classical world: emergence of trajectories in a quantum system Rodolfo Figari and Alessandro Teta

Propagation of chaos and effective equations in kinetic theory: a brief survey Mario Pulvirenti and Sergio Simonella

What decides the direction of a current? Christian Maes

A remark on eigenvalue perturbation theory at vanishing isolation distance Fiorella Barone and Sandro Graffi

Some results on the asymptotic behavior of finite connection probabilities in percolation Massimo Campanino and Michele Gianfelice

Correlation inequalities for the Potts model Geoffrey R. Grimmett

Quantum mechanics: some basic techniques for some basic models, I: The models Vincenzo Grecchi

Quantum mechanics: some basic techniques for some basic models, II: The techniques Vincenzo Grecchi

On stochastic distributions and currents

Vincenzo Capasso and Franco Flandoli

A note on Gibbs and Markov random fields with constraints and their moments Alberto Gandolfi and Pietro Lenarda

Quantum mechanics: light and shadows (ontological problems and epistemic solutions) Gianfausto Dell'Antonio

MEMOCS is a journal of the International Research Center for the Mathematics and Mechanics of Complex Systems at the Università dell' Aquila, Italy.

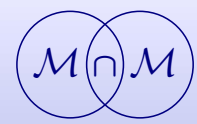

